\title{
Evaluation of analytical performance specifications of routine clinical biochemistry tests with biological variation- based total allowable error criteria
}

\author{
Orhan Cakmak', ¿ Zeynep Altun², i Nilhan Nurlu Ayan³
}

\author{
'Department of Medical Biochemistry, Mardin State Hospital, Mardin, Turkey \\ 2Department of Medical Biochemistry, Patnos State Hospital, Agrı, Turkey \\ ${ }^{3}$ Department of Medical Biochemistry, Health Sciences University Gaziosmanpasa Taksim Education and Research Hospital, Istanbul, Turkey
}

\begin{abstract}
Objectives: Total allowable error (TEa) is the analytical quality specification that determines the acceptable limits for a test result. The aim of the present study was to determine whether third-party control data (percentage coefficient of variation (CV), percentage bias, and \%TEa) of 26 clinical biochemistry parameters collected over a period of 6 months would meet biological variation (BV)-based analytical quality specifications (minimum, desirable, and optimum) in which BV quality specification might be more appropriate for our laboratory use.

Methods: The study was conducted for 6 months on tests with the TEa values determined according to BV and using third-party controls, Unity Real Time ${ }^{\circledast}$ software, and Beckman Coulter AU5800 clinical chemistry analyzer.

Results: The BV minimum specification is the easiest target to be performed by laboratories since it has the widest limits. It is also known that the BV already fulfills the other specifications (minimum and desirable) in tests where the optimum specification targets can be achieved, and the test performance classifications of our work are made according to this information. The minimum specification is only in Level 2 control serum total cholesterol test. The optimum specification with the narrowest limits within the BV criteria and the most difficult to achieve was met in nine tests (alanine aminotransferase, direct bilirubin, total bilirubin, creatine kinase, gamma-glutamyl transferase, ferritin, lipase, triglyceride, and uric acid (UA) for both control levels. Outside of these tests, in general, the desirable specification that each laboratory is aiming to fulfill is met.

Conclusion: It is very valuable to set the boundaries of quality specifications targeted for analytical performance based on BV. These values are determined by the intra-individual and inter-individual variability of the test and, thus, define a test-specific target. In addition to defining the TEa for each specification of BV, the definition of bias and CV boundaries can give a more objective idea of the source of error.
\end{abstract}

Keywords: Analytical performance, biological variation, total allowable error

$\mathrm{T}$ housands of results are produced in clinical laboratories using assay procedures. These results are used for many purposes, such as group screening, diagnosis, monitoring, and differential diagnosis of diseases. Many variations are encountered in total assay procedures in laboratories, including pre-analytical, analytical, and biological variations (BVs). In order to obtain reliable results, clinical laboratories should minimize all sources of variation and manage all the testing process well. This management is applicable for all types of variation except BV [1, 2].

Address for correspondence: Nilhan Nurlu Ayan, MD. Department of Medical Biochemistry, Health Sciences University Gaziosmanpasa Taksim Education and Research Hospital, Istanbul, Turkey

Phone: +90 2122524300 E-mail: nilhannurlu@yahoo.com ORCID: 0000-0002-0844-5050

Submitted Date: May 18, 2018 Accepted Date: July 12, 2018 Available Online Date: September 24, 2018

${ }^{\circ}$ Copyright 2018 by International Journal of Medical Biochemistry - Available online at www.internationalbiochemistry.com 
BV is defined as the random fluctuations of an analyte concentration around a homeostatic set point in an individual [3]. The application of BV data in the clinical laboratory is very comprehensive and valuable. The components of $\mathrm{BV}$ can be used to determine the timing and frequency of sampling in the pre-pre-analytical and pre-analytical phases; quality specifications, such as imprecision, bias, and total error in the analytical phase; and population-based reference intervals, individuality index, and reference change values in the post-analytical and post-post-analytical phases; they are very important during the interpretation and evaluation of test results $[2,4]$.

The aim of the present study was to determine whether thirdparty control data of 26 clinical biochemistry parameters collected over a period of 6 months would meet BV-based analytical quality specifications (minimum, desirable, and optimum). For this purpose, we decided to use third-party control samples independent of the manufacturers of the devices and reagents present in our laboratory. We procured control samples sufficient for a 1-year period. These samples met the required conditions in terms of stability and analyte concentration at clinical decision level, and so on. We used these control samples for internal quality control in our laboratory, and the results were evaluated by the Unity Real Time ${ }^{\circledR}$ program, which was used to compare results on an international scale, and the Westgard Advisor $^{\mathrm{TM}}$ software, which was an integral part of the program.

Using the Unity Real Time ${ }^{\circledR}$ program, the mean, standard deviation, and coefficient of variation (CV) values were calculated for the measurands evaluated using the internal quality assurance program.

Bias is defined as "the systematic deviation in the test results indicating the difference between the mean of the serial replicate measurements and the expected true value or the value that is accepted as true" [5]. The programs mentioned above enable us to compare our mean values as calculated for each test using peer-group means, which we accepted as the true value. Unlike the external quality assessment programs, in which a single measurement result is compared with the peer-group mean, these programs allowed us to detect our actual bias.

We investigated whether the $C V$ and bias values of each test and the total analytical error (TAE) derived from these values were within the CV, bias, and total allowable error (TEa) limits that are required by each of the three quality specifications of $\mathrm{BV}$ (minimum, desirable, and optimum), and also, which BV quality specification was more appropriate for use in our laboratory.

\section{Materials and Methods}

In the present study, test performances in Gaziosmanpasa Taksim Education and Research Hospital's Biochemistry Laboratory were evaluated according to the BV acceptance criteria (minimum, desirable, and optimum) as updated by Ricos and colleagues [6]. Furthermore, the quality specifications of BV that could be met by the performance of routinely analyzed biochemical tests were identified.
The study was conducted over a 6-month period from February 3 to August 3, 2016 using a Beckman Coulter AU5800 clinical chemistry analyzer. The study duration and the method to be used in data evaluation were both determined according to the Clinical Laboratory and Standards Institute (CLSI) C24-A3 guideline, which states that the mean, standard deviation, and control limits should be determined based on the cumulative data obtained over a period of 3 to 6 months [7].

During the study period, the preliminary data were collected over 20 working days in 1 month, with 40 measurements made for each level, and these data were used to calculate the preliminary mean and standard deviation values (Biorad Lypocheck Clinical Chemistry Control Level 1; lot no: 14461) and Level 2; lot no: 14462). The cumulative mean and standard deviation values were calculated by adding the new data to the initial values. Cumulative control limits are known to be a better indicator of long-term test performance [8].

For each test, the control rules and control measurement numbers were determined using the Westgard Advisor software according to the analytical CV and bias values of the tests. The Unity Real Time ${ }^{\circledast}$ software was set to detect control violations automatically. Thus, the control numbers and rules were applied during the process. The detected rule violations were subjected to the necessary intervention, and the interventions

Table 1. Test and methods in our laboratory

\begin{tabular}{lcc}
\hline Test Name & Test Unit & Method \\
\hline Albumin & $\mathrm{g} / \mathrm{dL}$ & Colorimetric BCG \\
ALT & $\mathrm{IU} / \mathrm{L}$ & $\mathrm{IFCC}$ (and/or PLP Activation) \\
ALP & $\mathrm{IU} / \mathrm{L}$ & IFCC AMP Buffer \\
Amylase & $\mathrm{IU} / \mathrm{L}$ & CNP-Trioside CNPG3 \\
AST & $\mathrm{IU} / \mathrm{L}$ & $\mathrm{IFCC}$ (and/or PLP Activation) \\
Direct Bilirubin & $\mathrm{mg} / \mathrm{dL}$ & Colorimetric Diazo method \\
Total Bilirubin & $\mathrm{mg} / \mathrm{dL}$ & Colorimetric Diazo method \\
Chlorid & $\mathrm{mEq} / \mathrm{L}$ & ISE indirect \\
Calcium & $\mathrm{mg} / \mathrm{dL}$ & Colorimetric Arsenoza III \\
HDL-Cholesterol & $\mathrm{mg} / \mathrm{dL}$ & Enzymatic immunoinhibition \\
Total Cholesterol & $\mathrm{mg} / \mathrm{dL}$ & CHO-POD \\
Creatinine kinase & $\mathrm{IU} / \mathrm{L}$ & Modified IFCC method \\
Creatinine & $\mathrm{mg} / \mathrm{dL}$ & Modified Jaffe's Kinetic \\
GGT & $\mathrm{IU} / \mathrm{L}$ & $\mathrm{G}-\mathrm{Glutamyl}$ carboxy nitroanylide \\
Glucose & $\mathrm{mg} / \mathrm{dL}$ & Hexokinase \\
Ferritin & $\mathrm{\mu g} / \mathrm{dl}$ & Colorimetric TPTZ \\
LDH & $\mathrm{IU} / \mathrm{L}$ & LDH (L-P) IFCC \\
Lipase & $\mathrm{IU} / \mathrm{L}$ & Enzymatic 1.2-diglyceride \\
Magnesium & $\mathrm{mEq} / \mathrm{L}$ & Xylidyl blue \\
Potassium & $\mathrm{mEq} / \mathrm{L}$ & ISE indirect \\
Phosphor & $\mathrm{mg} / \mathrm{dL}$ & Phosphomolybdate \\
Total proteine & $\mathrm{g} / \mathrm{dL}$ & Biuret \\
LDL-Cholesterol & $\mathrm{mg} / \mathrm{dL}$ & Enzymatic immunoinhibition \\
Sodium & $\mathrm{mEq} / \mathrm{L}$ & ISE indirect \\
Triglyceride & $\mathrm{mg} / \mathrm{dL}$ & GPO-POD \\
Uric acid & $\mathrm{mg} / \mathrm{dL}$ & Uricase \\
& &
\end{tabular}




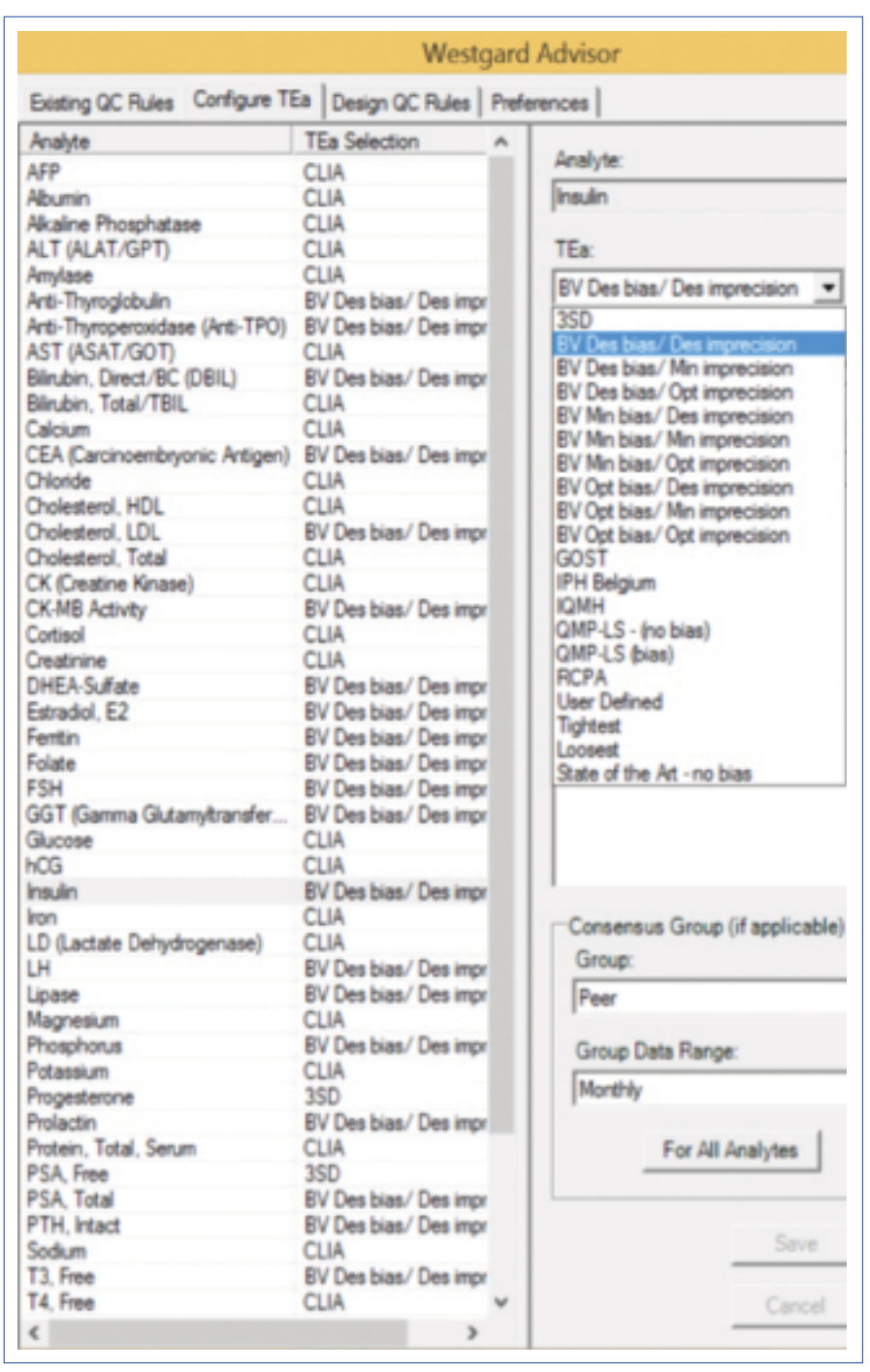

Figure 1. Determination of the TEa configuration for each test

were recorded. Percentage bias (\%B), percentage CV (\%CV), and TAE were calculated for 26 tests. We followed the policy that we established as the target for the Clinical Laboratory Improvement Amendments (CLIA) criteria for these tests in our laboratory and then made comparisons with the BV acceptance criteria. During the 6-month study period, performance reports of all tests were obtained separately according to CLIA and BV.

\section{Examined tests and assay methods}

All examined tests were analyzed using a Beckman Coulter AU5800 analyzer throughout the 6-month study period in accordance with the planned control procedure. The evaluation comprised 26 tests all analyzed using the routine clinical chemistry analyzer. Table 1 shows these tests, their units, and the assay methods.

\section{Quality control procedure}

As shown in Fig. 1, TEa for each test was determined according to BV specifications using the software program. The Westgard Advisor ${ }^{\mathrm{TM}}$ module, which was pre-defined in the program, the calculated $\% \mathrm{~B}, \% \mathrm{CV}, \Delta \mathrm{SE}$ (critical systematic error), and sigma ( $\sigma$ ) values for the desired parameters for a given time interval. Based on these data, the program automatically calculated the required control rule and control measurement number at the analytical quality assurance percentage, providing the best test performance according to the most appropriate error detection (Ped; probability of error detection) and false rejection (Pfr; probability of false rejection) values with the help of both OPSpecs and critical error graphics.

\section{Allowable error values based on BV specifications}

Table 2 shows the $\% \mathrm{TEa}, \% \mathrm{~B}$, and \%CV limits of the examined tests according to BV minimum, desirable, and optimum specifications.

All \%TEa, \%B, and \%CV values for the desired specifications were derived from the $\mathrm{BV}$ data. For minimum and optimum specifications, the $\% \mathrm{TEa}, \% \mathrm{~B}$, and \%CV limit values for the tests that did not have a specified value in the data were calculated using the formula given below [6]:

\section{For minimum specification:}

$\% \mathrm{CV}<0.75 \mathrm{CV}_{\mathrm{w}}$

$\% \mathrm{~B}<0.375\left(\mathrm{CV}_{\mathrm{w}}{ }^{2}+\mathrm{CV}_{\mathrm{b}}{ }^{2}\right)^{1 / 2}$

$\% \mathrm{TEa}<0.375\left(\mathrm{CV}_{\mathrm{w}}{ }^{2}+\mathrm{CV}_{\mathrm{b}}{ }^{2}\right)^{1 / 2}+1.65\left(0.75 \mathrm{CV}_{\mathrm{w}}\right)$

\section{For optimum specification:}

$\% \mathrm{CV}<0.25 \mathrm{CV}_{\mathrm{w}}$

$\% \mathrm{~B}<0.125\left(\mathrm{CV}_{\mathrm{w}}{ }^{2}+\mathrm{CV}_{\mathrm{b}}{ }^{2}\right)^{1 / 2}$

$\%$ TEa $<0.125\left(\mathrm{CV}_{\mathrm{w}}{ }^{2}+\mathrm{CV}_{\mathrm{b}}{ }^{2}\right)^{1 / 2}+1.65\left(0.25 \mathrm{CV}_{\mathrm{w}}\right)$

where:

\%TEa: total allowable error; a quality requirement for a given test,

$\% \mathrm{~B}$ : deviation from the true value,

$\% \mathrm{CV}$ : imprecision observed for a given method,

$\mathrm{CVw}$ : intra-individual biological variation,

CVb: inter-individual biological variation,

1.65: coefficient for $95 \%$ confidence interval.

For all tests, TAE, \%CV, and \%B were calculated, and the BV acceptance criteria (minimum, desirable, and optimum) were used for comparison. TAE for each level of each test was calculated using the TAE $=\% B+1.65 \times C V$ formula in the Excel program. The \%B value was accepted as its absolute value. 1.65 is the coefficient used for $95 \%$ confidence interval. The performance reports include the observed $\% \mathrm{~B}$, the $\% \mathrm{CV}$ values for the given level, and the recommended control rule and numbers based on the BV minimum, desirable, and optimum acceptance criteria.

\section{Results}

The tests using the Level 1 and Level 2 control materials that we assayed in the clinical biochemistry laboratory following 


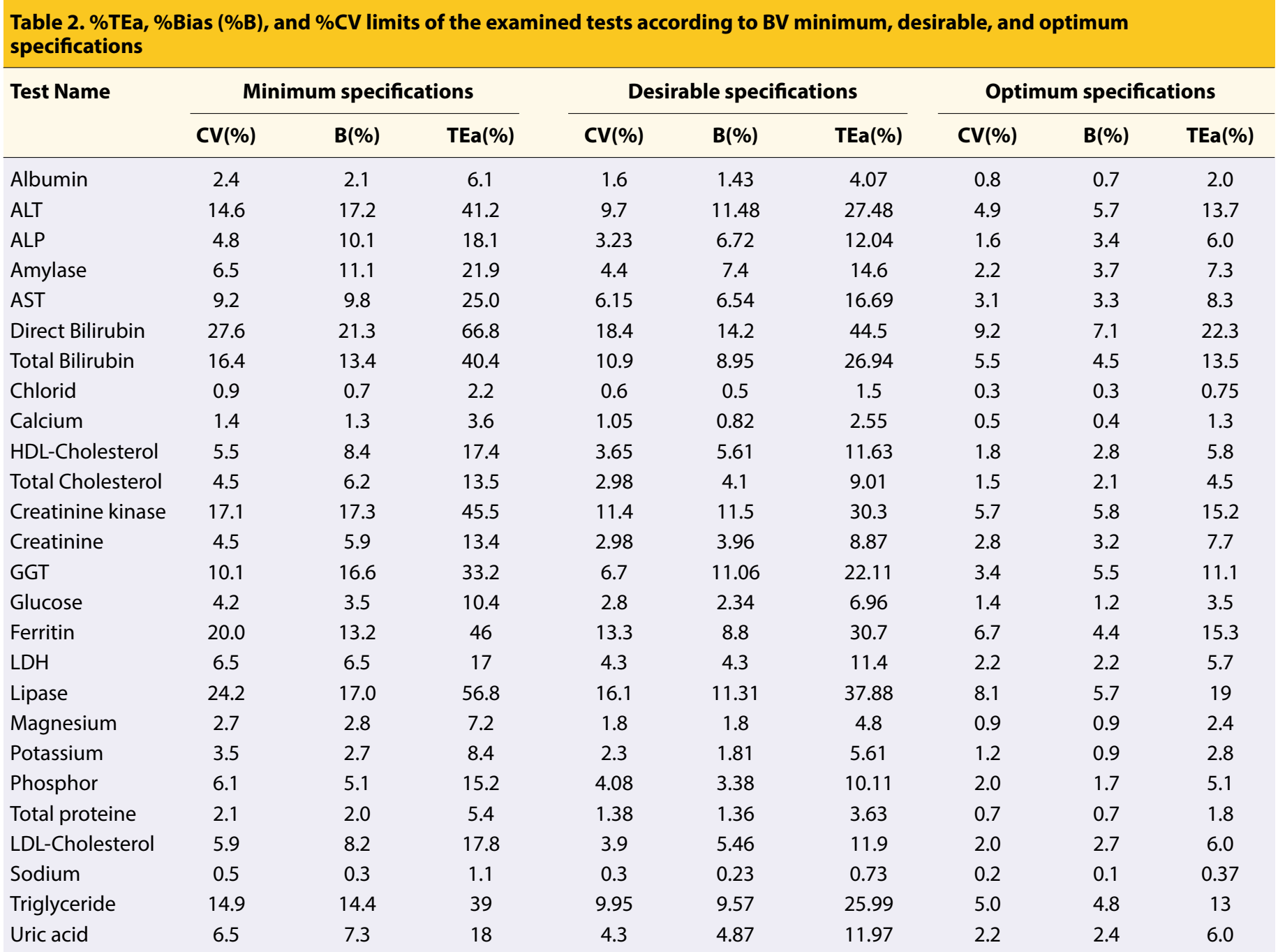

Table 3. Classification of tests in Level 1 and Level $\mathbf{2}$ control materials that met the BV TEa acceptance criteria (minimum, desirable, and optimum)

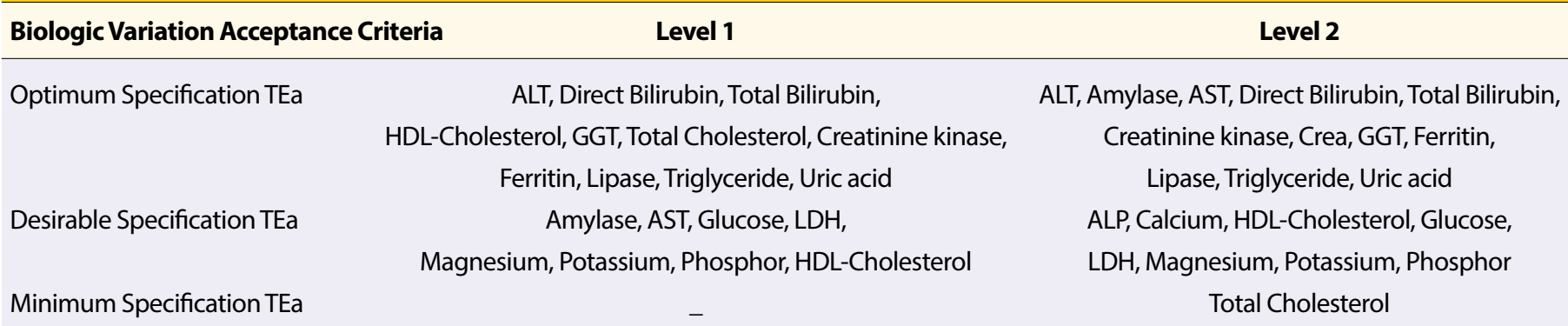

the CLIA protocols are presented in Table 3 according to the BV TEa acceptance criteria (minimum, desirable, and optimum) that they met. Table 4 shows the Level 1 and 2 control material tests that met the BV optimum acceptance criteria. Table 5 shows the tests that did not meet any BV acceptance criteria. Although the analytical CV and/or bias for our laboratory was higher than the allowable $\mathrm{CV}$ and/or bias based on BV for both control levels, we principally targeted $T A E<T E a$ based on the BV criteria, and all the evaluations were made accordingly.
In the present study, CVw, CVb, and the allowable $C V$ values derived from these values were obtained from the BV data. For the Level 1 control material, 11 tests (alanine aminotransferase $(A L T)$, direct bilirubin (DB), total bilirubin (TB), high-density lipoprotein cholesterol (HDL-Chol), total cholesterol (T-Chol), creatine kinase (CK), gamma-glutamyl transferase (GGT), ferritin, lipase (Lip), triglyceride (Tg), and uric acid (UA) met the BV optimum, and 8 tests (amylase (Amy), aspartate aminotransferase (AST), glucose (Glu), lactate dehydrogenase (LDH), 


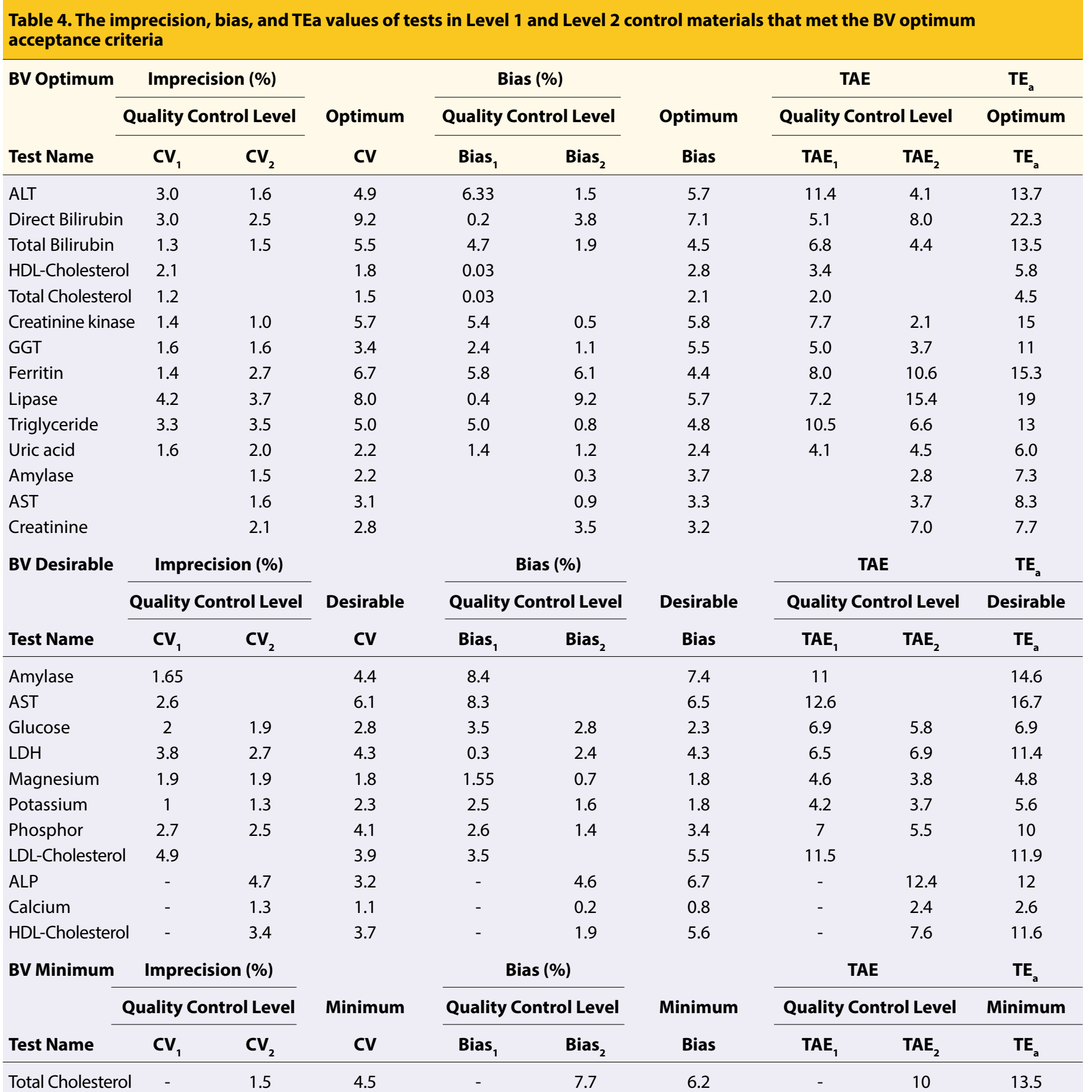

magnesium (Mg), potassium (K), phosphor (P), and low-density lipoprotein cholesterol (LDL-Chol) met the BV desirable TEa criteria. Seven tests (albumin (Alb), alkaline phosphatase $(\mathrm{ALP})$, chloride $(\mathrm{Cl})$, calcium (Ca), creatinine (Crea), total protein (TP), and sodium (Na)) did not meet any BV acceptance criteria. For the Level 2 control material, 12 tests (ALT, Amy, AST, DB, TB, CK, Crea, GGT, Fe, Lip, Tg, and UA) met the BV optimum, 8 tests (ALP, Ca, HDL-Chol, Glu, LDH, Mg, K, and P) met the BV desirable, and 1 test (T-Chol) met the BV minimum TEa criteria.
Four tests (Alb, Cl, TP, and $\mathrm{Na}$ ) did not meet any BV acceptance criteria.

In our study, the Alb, Cl, TP, and Na parameters showed unacceptable performance (TAE>TEa) for both control levels. Additionally, the test performances of ALP, Ca, and Crea were outside the acceptable limits (TAE>TEa) for Level 1 control material.

When compared with other specification values, the optimum specification TEa, CV, and bias values have the narrowest range and are the most difficult of all BV specifications to achieve. 
Table 5. Tests that did not meet any BV acceptance criteria according to quality control levels

\begin{tabular}{|c|c|c|c|c|c|c|c|c|c|}
\hline \multirow{2}{*}{$\begin{array}{l}\text { BV Minimum } \\
\text { Test Name }\end{array}$} & \multicolumn{2}{|c|}{$\frac{\text { Imprecision (\%) }}{\text { Quality Control Level }}$} & \multirow{2}{*}{$\begin{array}{l}\text { Minimum } \\
\text { CV }\end{array}$} & \multicolumn{2}{|c|}{$\frac{\text { Bias (\%) }}{\text { Quality Control Level }}$} & \multirow{2}{*}{$\begin{array}{c}\text { Minimum } \\
\text { Bias }\end{array}$} & \multicolumn{3}{|c|}{ TAE } \\
\hline & $C V_{1}$ & $\mathrm{CV}_{2}$ & & Bias $_{1}$ & Bias $_{2}$ & & TAE $_{1}$ & $\mathrm{TAE}_{2}$ & $\begin{array}{c}\text { Minimum } \\
\text { TAE }_{a}\end{array}$ \\
\hline Albumin & 2.0 & 1.7 & 2.4 & 5.8 & 3.7 & 2.1 & 9.0 & 6.6 & 6.1 \\
\hline Chlorid & 0.9 & 1.1 & 0.9 & 2.1 & 0.5 & 0.7 & 3.6 & 2.4 & 2.2 \\
\hline ALP & 4.9 & - & 4.8 & 10.5 & - & 10 & 18.6 & - & 18.1 \\
\hline Calcium & 1.2 & - & 1.4 & 1.7 & - & 1.3 & 3.7 & - & 3.6 \\
\hline Creatinine & 2.7 & - & 4.5 & 12.9 & - & 5.9 & 17.3 & - & 13.4 \\
\hline
\end{tabular}

Table 6. Recommended quality control procedure for Level 1 and 2 quality control tests meeting the BV optimum specifications

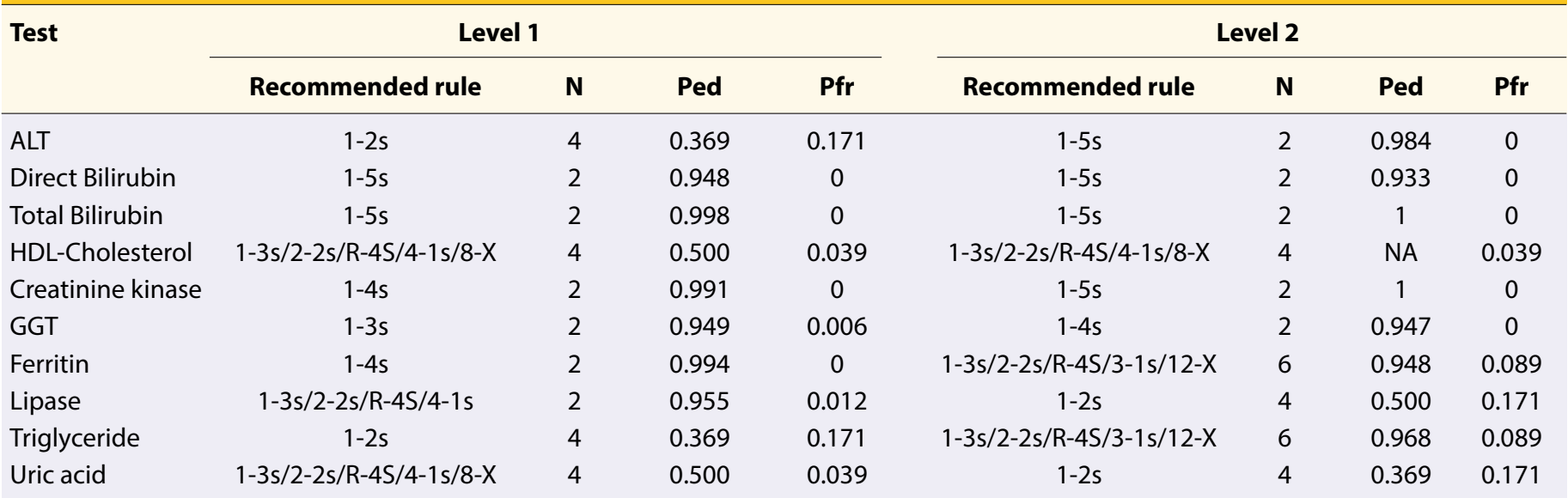

Table 6 shows the recommended quality control rule(s), the required number of quality control measurements $(N)$, the Ped values, and the Pfr values for the tests that meet these criteria to maintain test performance.

\section{Discussion}

In the present study, the results of the routine clinical biochemistry tests for Level 1 and Level 2 quality control materials were evaluated for performance according to the BV (minimum, desirable, and optimum) TEa acceptance criteria. In addition, the study aimed to determine the accuracy, precision, and reliability of the examined tests; to document sustainability of quality; and to improve quality.

Physicians request laboratory tests for approximately $80 \%$ of patients presenting to the hospital. Considering this, it is very important to prevent errors by improving the quality of each testing procedure. Problems, such as repeated test requisitions, unnecessary therapeutic interventions, and wasted money, time and labor, plus distrust in the laboratory can all be avoided by improving the quality of the total testing process (TTP) [9].

Laboratory managers have the responsibility of detecting the sources of error impeding the TTP and correcting them. Ana- lytical errors account for $21 \%$ of all errors occurring in the TTP, but they may be easier to manage compared with pre-analytical or post-analytical errors $[10,11]$. This is because quality control procedures and several quality planning tools have been devised for analytical quality management.

Three different quality specifications exist according to BV for the evaluation of analytical quality performance. Since BV minimum specification (min. imprecision/min. bias) has the widest range, it is regarded as the easiest target for laboratories to achieve. Furthermore, BV desirable specification (des. imprecision/des. bias) is a target for every laboratory in general. BV optimum specification (opt. imprecision/opt. bias) has the narrowest range and is the most difficult to achieve. When BV optimum specification targets are met, other specifications (minimum and desirable) are already achieved. Similarly, when BV desirable specification targets are met, BV minimum targets are already achieved. The performance test classifications used in the present study are based on this fact [12]. It is considered best for our laboratory to maintain the TEa target for these tests according to BV optimum specifications and according to BV desirable specifications when possible. For those tests that only meet BV minimum specifications or do not meet any BV-based criteria, we aim to develop and im- 
plement the necessary procedures to at least achieve the BV desirable specification criteria.

In the present study, we targeted the more stringent, BVbased analytical quality goals instead of the wider analytical quality targets (e.g., CLIA and Turkey). While it is easier to meet the general quality specifications with the existing methodology and technology for some tests, such as Tg using the threestage BV model [3], which is derived from imprecision, bias, and total error limits in BV, it may be harder for other tests, such as $\mathrm{Ca}, \mathrm{Na}$, and $\mathrm{Cl}$.

Bal et al. [13] calculated imprecision values for 10 clinical biochemistry parameters using internal and external quality control data from three analyzers of the same brand and compared these values with the \%TEa values for Fraser and CLIA. The Alb, Na, TP, and Crea tests did not meet the BV-based quality specifications in any of the three devices, and this was explained by low target TEa limits. Similarly, in our study, while Alb, $\mathrm{Na}, \mathrm{TP}$, and $\mathrm{Cl}$ tests were able to meet the CLIA TEa targets, the BV TEa targets could not be achieved.

Studies have reported that it is quite difficult to meet the BV TEa criteria for electrolytes that are analyzed via the indirect ion selective electrode method, such as $\mathrm{Na}$ and $\mathrm{Cl}[14,15]$. Indeed, we were not able to meet the BV TEa criteria for these electrolytes in our study. Regardless, it may be possible to achieve the desired targets for these parameters by meticulously checking various details, such as inspecting the cleaning system, following a stringent maintenance protocol, and checking the water purifying systems and consumables.

In our study, different TAE values were obtained for the two levels of control samples depending on the analyte concentrations. BV TEa quality specifications could not be achieved in seven tests for Level 1 control materials and four tests for Level 2 control materials. For instance, Level 1 control results for the ALP and Ca test did not meet the minimum BV-based TEa criteria. One study, which applied Six Sigma to evaluate the performance of analytical phase and calculated bias values from external quality assurance results over a 6-month period, found the sigma values of the internal quality sample for the ALT test to be 2.79 for the normal level and 4.24 for the pathological level [16].

Although calculation of the CV and TAE values for the same analyte at different concentrations is cumbersome and costly, it allows for a more reliable assessment of that analyte's performance. In our study, we calculated the monthly bias values using the daily mean peer-group values of the inter-laboratory internal quality control comparison program. Such an assessment of bias allows any deviation to be noticed earlier than the reported bias in external quality control results. Therefore, we believe that it is very useful and reliable for developing prospective corrective actions.

In their study, Coskun et al. [17] presented their internal and external quality assessment using control samples of the same matrix, and they reported that $\mathrm{T}-\mathrm{Chol}, \mathrm{Glu}, \mathrm{Ca}, \mathrm{Cl}, \mathrm{K}, \mathrm{Mg}, \mathrm{Na}$, and GGT for Level 1 control material and T-Chol, Glu, UA, Crea,
$\mathrm{Ca}, \mathrm{Cl}, \mathrm{Na}$, and $\mathrm{CK}$ for Level 2 control material do not meet any BV TEa criteria. Their results are consistent with the results of the present study.

In another study, Fernandez-Calle et al. [15] performed a validation assessment of the two new clinical chemistry analyzers brought to their laboratory. For this purpose, they calculated the $\mathrm{CV}, \% \mathrm{~B}$, and total error values of 30 analytes, and they chose the BV criteria as their target. Similar to our study, they used the Bio-Rad control materials and Unity Real Time software, and they used the cumulative peer-group mean from the third-party controls as the reference value when evaluating bias. For each test, the BV criteria were determined (minimum, desirable, and optimum), and bias and imprecision were evaluated separately. The authors emphasized that the control materials that are to be used in such studies should have a matrix similar to the patient samples and should contain an analyte concentration at clinical decision level according to the CLSI guidelines. In their BV-based ISO 15189 laboratory accreditation study, the $\mathrm{Na}, \mathrm{Cl}$, and $\mathrm{Mg}$ tests did not meet the TEa criteria in either the Level 1 or Level 2 control materials. Although the Alb, ALP, Ca, Crea, Glu, K, and P tests were able to meet the TEa criteria in that study, these tests did not meet any of the bias or CV values.

Despite some weaknesses, the TAE methods are widely used for performance calculations in laboratory medicine. Regardless, other areas of metrology have shifted to the measurement uncertainty (MU) methods. The Guide to Expression of Uncertainty in Measurements (GUM) and the International Vocabulary of Metrology (VIM) do not use the concept of "true value." However, the TAE methods are based on the concept of "true value." The closer the true value of the measurement result is, the smaller the error that will occur. By contrast, $\mathrm{MU}$ is not based on "true value" $[18,19]$.

When evaluating analytical performance, the uncertainty of the whole test process must be included in the account. The analytical variation constitutes only a part of the total uncertainty. BV, pre- and post-analytical variation all need to be included. MU includes these variations, but there are few instances of information on the causes of these variations being applied to the MU theory. According to the GUM, the MU methods in laboratory medicine have not developed sufficiently to cope with the diagnostic uncertainty. The development of analytical performance characteristics for diagnostic uncertainty has resulted in qualitative improvement. Today, the error and uncertainty methods are used as complementary methods when evaluating measurement results in laboratory medicine $[20,21]$.

\section{Conclusion}

Determining $C V$, bias, and TEa limits for the targeted analytical performance quality specifications based on BV is very valuable. Since these values are determined depending on the "within-individual" and "between-individual" variations of the 
test, they define a target that is specific for the test. Moreover, defining allowable bias and CV limits separately for each specification of BV in addition to TEa evaluation can give a more objective idea about the source of error.

Conflict of interest: There is no conflict of interest between the authors.

Fundings: Gaziosmanpasa Taksim Education and Research Hospital.

Peer-review: Externally peer-reviewed.

Authorship contributions: Concept - N.N.A., Z.A., O.C.; Design N.N.A., O.C.; Supervision - N.N.A., O.C.; Materials - Z.A., O.C.; Data collection \&/or processing - Z.A., O.C.; Analysis and/or interpretation - O.C., Z.A., N.N.A.; Literature search - O.C., Z.A., N.N.A.; Writing - O.C., N.N.A.; Critical review - N.N.A.

\section{References}

1. Ricós C, Perich C, Minchinela J, Álvarez V, Simón M, Biosca C, et al. Application of biological variation - a review. Biochemia Medica 2009;19:250-9. [CrossRef]

2. Fraser CG, Harris EK. Generation and application of data on biological variation in clinical chemistry. Crit Rev Clin Lab Sci 1989;27:409-37. [CrossRef]

3. Fraser CG. Biological Variation: From Principles to Practice. Washington: AACC Press; 2001. p. 11-6.

4. Petersen $\mathrm{PH}$, de Verdier $\mathrm{CH}$, Groth $\mathrm{T}$, Fraser $\mathrm{CG}$, Blaabjerg $\mathrm{O}$, Hørder M. The influence of analytical bias on diagnostic misclassifications. Clin Chim Acta 1997:260;189-206. [CrossRef]

5. Burtis CA, Bruns DE. Selection and Analytical Evaluation of Methods - With Statistical Techniques. In: Tietz Fundamentals of Clinical Chemistry and Molecular Diagnostics. 7th ed. Amsterdam: Elsevier; 2015. p. 6-31.

6. Perich $C$, Minchinela J, Ricós $C$, Fernández-Calle $P$, Alvarez V, Doménech $\mathrm{MV}$, et al. Biological variation database: structure and criteria used for generation and update. Clin Chem Lab Med 2015;53:299-305. [CrossRef]

7. CLSI C24A3: Statistical Quality Control for Quantitative Measurement Procedures: Principles and Definitions; Approved Guideline, C24-A3. 3th ed. Wayne: Clinical and Labaratory Standards Institute; 2006.

8. Westgard J. Basic QC Practices. Training in Statistical Quality Control for Medical Laboratories. 3rd ed. Westgard QC; 2010. p. $175-89$.

9. Aslan D, Sert S, Aybek H, Yılmaztürk G. Assessment of Total Clinical Laboratory Process Perfromance: Normalized OPSpecs
Charts, Six Sigma and Patient Test Results [Article in Turkish]. Turk J Biochem 2005;30:296-305.

10. Stroobants AK, Goldschmidt HMJ, Plebani M. Error budget calculations in laboratory medicine: linking the concepts of biological variation and allowable medical errors. Clin Chim Acta 2003;333:169-76. [CrossRef]

11. Fuentes-Arderiu X, Acebes-Frieyro G, Gavaso-Navarro L, Castiñeiras-Lacambra MJ. Pre-metrological (pre-analytical) variation of some biochemical Quantities. Clin Chem Lab Med 1999;37:987-9. [CrossRef]

12. Fraser CG, Hyltoft Petersen P, Lieeber JC, Ricos C. Proposals for setting generally applicable quality goals solely based on biology. Ann Clin Biochem 1997;34:8-12. [CrossRef]

13. Bal C, Serdar MA, Güngör OT, Çelik HT, Abuşoğlu S, Uğuz N, et al. Calculation of measurement uncertainty of biochemical parameters [Article in Turkish]. Turk J Biochem 2014;39:53843.

14. Ricós C, Ramón F, Salas A, Buño A, Calafell R, Morancho J, et al; Interdisciplinary Expert Committee for Quality Specifications in Clinical Laboratory. Minimum analytical quality specifications of inter-laboratory comparisons: agreement among Spanish EQAP organizers. Clin Chem Lab Med 2011;50:45561.

15. Fernandez-Calle $P$, Pelaz $S$, Oliver P, Alcaide MJ, Gomez-Rioja $\mathrm{R}$, Buno $A$, et al. The importance of having a flexible scope ISO 15189 accreditation and quality specifications based on biological variation-the case of validation of the biochemistry analyzer Dimension Vista. Biochem Med (Zagreb) 2013;23:83-95.

16. Ercan Ş. The Evaluation of Analytical Phase According to Six Sigma [Article in Turkish] Türk Klinik Biyokimya Derg 2015;13:59-68.

17. Coskun A, Ibrahim Unsal I, Serteser M, Fraterman A. Toward standardization of quality assessment in laboratory medicine by using the same matrix samples for both internal and external quality assessments. Accred Qual Asur 2009;14:547-52.

18. Westgard JO. Useful measures and models for analytical quality management in medical laboratories. Clin Chem Lab Med 2016;54:223-33. [CrossRef]

19. Oosterhuis WP, Theodorsson E. Total error vs. measurement uncertainty: revolution or evolution? Clin Chem Lab Med 2016;54:235-9. [CrossRef]

20. Oosterhuis WP, Bayat $H$, Armbruster D, Coskun A, Freeman KP, Kallner $A$ et al. The use of error and uncertainty methods in the medical laboratory. Clin Chem Lab Med. 2018;56:209-19.

21. Panteghini M, Sandberg S. Total error vs. measurement uncertainty: the match continues. Clin Chem Lab Med. 2016;54:1956. [CrossRef] 